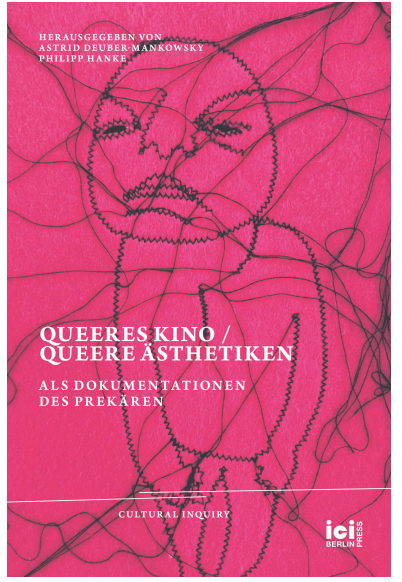

Queeres Kino / Queere Ästhetiken als Dokumentationen des Prekären, hg. v. Astrid Deuber-Mankowsky und Philipp Hanke, Cultural Inquiry, 22 (Berlin: ICI Berlin Press, 2021), S. 97-117

\section{HENRIETTE GUNKEL}

\section{Codes, Raster, Technologien queerer Erinnerungslandschaften} Charlotte Prodgers SaFO5

\author{
ZITIERVORGABE:
}

| Henriette Gunkel, >Codes, Raster, Technologien queerer Erinnerungslandschaften. Charlotte Prodgers $\mathrm{SaFO5} \ll$, in Queeres Kino / Queere Ästhetiken als Dokumentationen des Prekären, hg. v. Astrid Deuber-Mankowsky und Philipp Hanke, Cultural Inquiry, 22 (Berlin: ICI Berlin Press, 2021), S. 97-117<https://doi.org/10. 37050/ci-22_05>

\section{ANGABE ZU DEN RECHTEN:}

(C) bei den Autor*innen

Dieses Werk ist veröffentlicht unter einer Creative Commons Namensnennung - Weitergabe unter gleichen Bedingungen 4.0 International Lizenz.

ABSTRACT: Dieser Beitrag fokussiert die Arbeiten von Charlotte Prodger als ein Beispiel für gegenwärtige künstlerische Strategien, die sich bewusst einer Vereinnahmung bzw. einer Kommodifizierung queerer Ästhetiken verweigern und stattdessen die Notwendigkeit einer queeren Selbstbestimmung und damit einer queeren Bewegungsgeschichte (erneut) in den Vordergrund rücken. Insbesondere der 2019 bei der Biennale in Venedig gezeigte Film SaF05 wird dabei im Zentrum der Überlegungen stehen - eine Arbeit, die in gewisser Weise an experimentelle Prozesse des New Queer Cinema anknüpft und die Betrachtenden in und durch queere (Erinnerungs-)Landschaften führt.

SCHLAGWÖRTER: mediated landscapes; Transformation audiovisueller Medien; land art; Praktiken des Attunements; Autobiografie; Löwin; queere Selbstbestimmung; queere Ästhetik 


\section{Codes, Raster, Technologien queerer Erinnerungslandschaften \\ Charlotte Prodgers SaF05 \\ HENRIETTE GUNKEL}

Charlotte Prodgers 39-minütiger Einkanal-Videofilm SaF05 von 2019 beginnt mit einer Einstellung in schwarz-weiß. ${ }^{1} \mathrm{Zu}$ sehen sind die Hinterbeine und der Schwanz eines Löwen oder einer Löwin. Das Raubtier steht auf einer Lichtung; im Hintergrund sind trockenes Gras und Büsche zu sehen. Der abgebildete Kamerarahmen verweist darauf, wann die Aufnahme gemacht wurde: am 07.07.2017 um 6:34:50 Uhr morgens, bei sechs Grad. Allein der diegetische Sound sowie das kaum wahrnehmbare Heben und Senken des Brustkorbes und der fortlaufende Timecode verweisen darauf, dass kein Standbild, sondern ein Bewegtbild zu sehen ist (s. Abb. 1). Um 6:35:12 gibt es einen ersten Schnitt; der Timecode springt auf 6:35:44, just zu dem Moment, in dem sich das Tier niederlegt. Zu sehen ist jetzt nur noch das linke hintere Bein und ein Rückenansatz - hier kommen zu den vorherigen Atembewegungen die Bewegungen von kleinen fliegenden Insekten zum Bild hinzu, die sich um das Licht der Kamera sammeln. Bei

1 SaF05, Regie: Charlotte Prodger (Hollybush Gardens, Charlotte Prodger, 2019). Ein-Kanal-Video mit Sound, 39 min. Edition of $4+1$ AP. Ich möchte den Herausgeber*innen sowie Ulrike Bergermann und Conny Mosley für die wichtigen und inspirierenden Anmerkungen zum Text danken. 


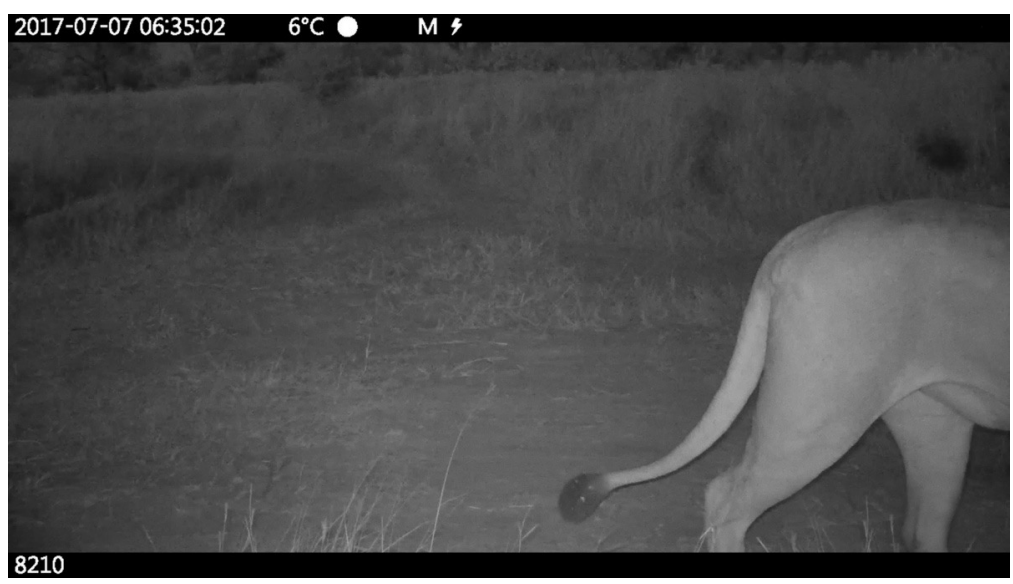

Abb. 1. Film-Still, SaF05, Regie: Charlotte Prodger (Copyright Hollybush Gardens, Charlotte Prodger, 2019).

6:36:12 folgt ein zweiter Schnitt. Das Tier, dessen Vorderseite noch immer nicht zu sehen ist, beginnt um 6:38:32 sich aus dem Kamerafokus herauszubewegen. Mittlerweile sind es sieben Grad. $\mathrm{Zu}$ sehen ist die Raubkatze bis zum nächsten Schnitt nicht mehr, aber zu hören: Langsam steigert sie sich in ein Brüllen (roaring), das als Zeichen der Dominanz und Markierung des Raumes mit männlichen Löwen assoziiert wird.

In den darauffolgenden Einstellungen, beginnend am 01.09.2018 um 22:00:57 bei $15 \mathrm{Grad}$, ist die Raubkatze dann in Gänze zu sehen. Das Tier, das uns von mindestens zwei unterschiedlichen fest installierten Kameras mit Bewegungsmeldern gezeigt wird, hat eine kleine (männlich kodierte) Mähne. Der Mähnenansatz ist mit dem eines jungen Löwen vergleichbar; allein das Alter des Tiers in Relation zu der Mähne scheint den Rückschluss zuzulassen, dass es sich bei den Aufnahmen um eine Löwin handelt. Gleichzeitig wird in der Ankündigung des Films SaF05, so der Name der Löwin, als eine der insgesamt fünf Löwinnen eingeführt, die im Okavango Delta männlich kodierte Körperausprägungen und Verhaltensweisen (Brüllen, Besteigen von anderen Löwinnen und das Markieren des Reviers) aufweisen, von denen SaF05 jedoch scheinbar die letzte Verbliebene ist. Diese Informationen leiten das Schauen des Films an, aber auch das Schreiben über den Film. SaF05 liegt auf der Lichtung, die Augen reflektieren 


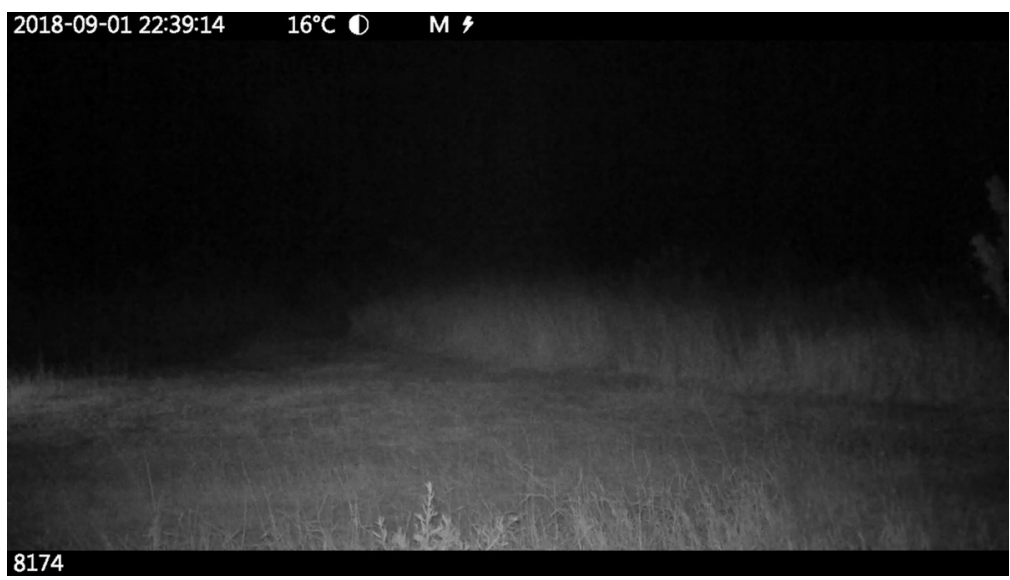

Abb. 2. Film-Still, SaF05, Regie: Charlotte Prodger (Copyright Hollybush Gardens, Charlotte Prodger, 2019)

das Licht der Kamera. Um 22:38:01 steht die Löwin gemächlich auf, macht einen leichten Buckel zur Dehnung und beginnt zu brüllen, indem sie den Kopf jeweils lang nach vorne zieht. Um 22:38:16 gibt es wieder einen Schnitt, auf eine andere Kamera, gleiches Datum, 22:39:02, 16 Grad. SaF05 läuft kurz links unten mit dem Kopf ins Bild, bleibt stehen, guckt und lauscht und dreht sich dann um 22:39:12 wieder links herum aus dem Bild. Die Kamera filmt weiter, und Prodger nimmt noch weitere 16 Sekunden mit in ihren Film (s. Abb. 2).

Wie die ersten Minuten zeigen, geht es in dem Film unter anderem um die Löwin SaF05, der Prodger im südlichen Afrika nachspürt - der sie aber nur durch das Filmmaterial aus Kamerafallen näher kommt, das Prodger vom Botswana Predator Conservation Trust (BPCT) für ihren Film zur Verfügung gestellt wurde. ${ }^{2}$ In den Ankündigungstexten zu SaF05 wird die Löwin als eine Art cipher verstanden, eine Wei-

2 Wie Linsey Young schreibt, reiste Prodger im November 2018 mit einem Dokumentarfilmteam nach Botswana, auf der Suche nach SaF05. Zu dem Zeitpunkt war die Löwin nicht mehr der Fokus eines Forschungsprojekts, weswegen es kaum Informationen zu ihrem Aufenthaltsort gab. Wie am Ende des Films zu hören, hat Prodger vor Ort mit dem BPCT gearbeitet, vor allem mit Bajaki Jacks Amos, dessen Stimme am Ende im Gespräch mit seinen Kolleg*innen über Funk zu hören ist. Auch weil Prodger und die unterschiedlichen Suchtruppen SaF05 nicht zu Gesicht bekommen, verschiebt sich Prodgers visueller Fokus auf Termitenhügel, während durch alte Logbucheinträge weiter von SaF05 in den Einsprechungen berichtet wird. Vgl. Linsey Young, »Hidden 
se, queere Zuneigung und Begehren zu denken (»a way of thinking about queer attachment and desire $\ll) .{ }^{3}$ Eine Denkfigur, die es erlaubt, über prekäre queere Verkörperungen nachzudenken, aber es geht in dem Film auch um die Landschaft, in der sich SaF05 bewegt, um die audio-visuelle Atmosphäre vor Ort - und um die Rahmung durch die Kameras.

Dem Fokus auf die Landschaft des Okavango Delta folgt ein erster Bruch im Film, eingeleitet durch ein Schwarzbild, das eine halbe Minute lang zu sehen ist, begleitet von Geräuschen des Wassers und des Windes, der gegen das Mikrophon von Prodgers iPhone presst. Das Wassergeräusch geht in ein neues, jetzt farbiges Bild über: eine vertikale Aufnahme/Perspektive, die von oben mit dem Smartphone die Spitzen von Prodgers Turnschuhen auf Gesteinsformationen an der Küste der griechischen Insel Paxos aufnimmt (s. Abb. 3), gefolgt von einem plötzlichem 90 Grad-Schwenk, der den Unterschied zwischen der vorherigen Standkamera (camera trap) und den Möglichkeiten des iPhones nicht deutlicher aufmachen könnte. Die Kamera verweilt jetzt auf weißem Gestein mit interessanter Struktur, leichter Färbung, Rissen und Abbröckelungen. Das Wasser, das die Gesteinsküste der Insel umspielt, ist weiterhin zu hören, fast meditativ, bevor es zu einem Schnitt auch auf der akustischen Ebene kommt. Der Sound von Dudelsackpfeifen ertönt, der allein durch die Lautstärke im starken Kontrast zum vorangehenden Sound steht und über diesen gelegt wird. Gleichzeitig wird der Begriff $\gg$ REVELATIONS « eingeblendet, eine Art Titel für ein Unterkapitel, das dem Film eine Struktur sowie eine inhaltliche Ausrichtung zu verleihen scheint. Die vertikale Perspektive wird auch nach dem Abwenden von der Gesteinsformation beibehalten; in einer ununterbrochenen Aufnahme von oben sehen

in Plain Sight «, in SaF05: Charlotte Prodger (Cove: Cove Park, Scotland + Venice, 2019), S. 11-17, hier S. 14-15.

$3 \gg$ SaF05 «, Ankündigung, Künstler*innenagentur LUX Scotland (2019) <https://lux. org.uk/work/saf05> [Zugriff: 2. Juni 2021]. Die Löwinnen-Sequenz ist wie ein Prelude, das dem Film den Titel gibt und ihn thematisch rahmt (Körper, Landschaften, Geschlecht), aber auch eine Verbindung zum vorherigen Film BRIDGIT, Regie: Charlotte Prodger (Hollybush Gardens, Charlotte Prodger 2016) aufmacht. Wie Astrid Deuber-Mankowsky schreibt, ist die Löwin bereits in BRIDGIT zu sehen, und zwar als Druck auf einem T-Shirt, das bei Prodger zu Hause über der Heizung hängt. Dieser Druck kann sich sowohl auf SaF05 als auch auf Bridget, eine Löwin mit Mähne im Oklahoma City Zoo, beziehen. Vgl. den Beitrag von Astrid Deuber-Mankowsky in diesem Band. 


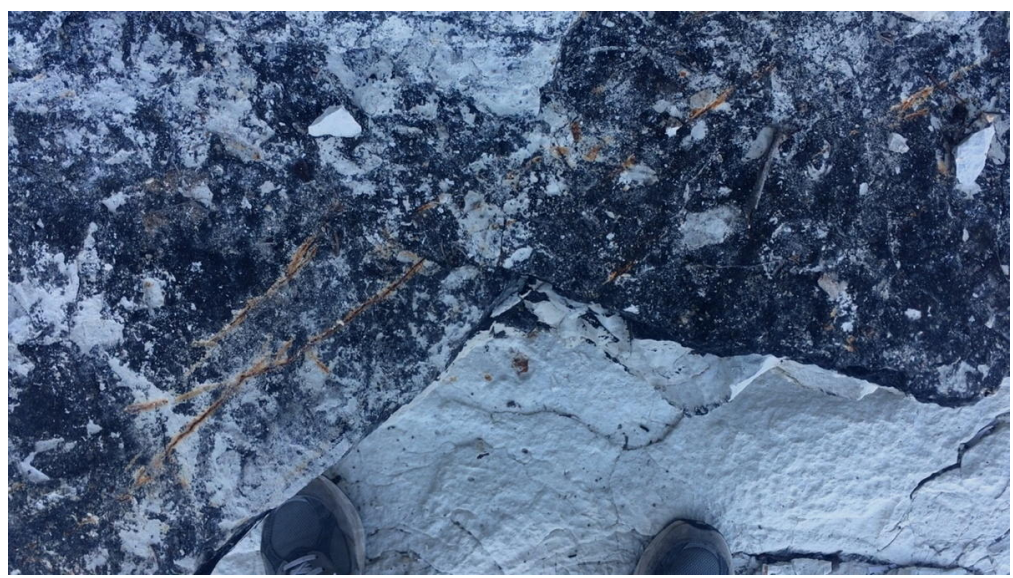

Abb. 3. Film-Still, SaF05, Regie: Charlotte Prodger (Copyright Hollybush Gardens, Charlotte Prodger, 2019).

wir Prodgers Beinen beim Laufen und leichtem Anstieg zu, bevor die Kamera wieder innehält und Ausschnitte der Gesteinsoberfläche einfängt, die $\gg$ rock strata, these layers upon layers of sedimentary rock all on top of each other «. ${ }^{4}$ Prodger hält die Kamera dabei nie ganz still, dann läuft sie wieder weiter, die Bewegung der Kamera wirkt leicht desorientierend, fast schwindelerregend.

Prodger situiert sich somit selbst in einer Umgebung, die eine andere ist als die von SaF05, und zieht doch visuelle und narrative Verbindungen zwischen beiden. Ihre Stimme aus dem Off, die über die Bilder der Küstenlandschaft von Paxos gelegt wird, beginnt mit den Worten: $\gg \mathrm{I}$ am thinking about a woman on TV in the last days of Pompeii. I am trying to wake up as a boy «. Was folgt, ist eine anekdotische Geschichte über die Zeit in einer sonntäglichen Bibelstundengruppe in Drumoak, Schottland, der sie aus Langeweile und wegen ihrer einzigen Freundin beitrat, als sie ungefähr zwölf Jahre alt war, und in der sie vor allem The Book of Revelation (das Buch der Offenbarung, das letzte Buch des Neuen Testaments) liest. Prodger erzählt, wie während eines Youth Fellowships eine Gruppe von Jungen aus Durris zu Besuch kommt, um der Bibelgruppe Breakdance beizu-

4 Charlotte Prodger on her Series Palace Prints (Hollybush Gardens, 2019) <https:// www.instagram.com/tv/B_qA9M8FtCJ/> [Zugriff: 2. Juni 2021]. 
bringen, und wie sie dem Ältesten unter ihnen beim Rauchen zusieht: $\gg[\mathrm{T}] \mathrm{o}$ tap the ash, he flicks the filter in staccato with his indexfinger. He does it more than necessary. It's repeated often like the spitting. Tapping and spitting take turns. « Dabei sieht man sie weiter über karge Gesteinsformationen gehen, sich von einer zur anderen bewegen, mit kurzen Momenten dazwischen, in der sie auf einer bestimmten Oberfläche verweilt. Das Beobachten der Gesteinsformationen bzw. der Landschaft verbindet sich mit dem Beobachten der Performativität von Geschlecht, wie bereits vorher bei SaF05, in diesem Fall bei den männlich kodierten Gesten des Rauchens. Die Landschaft in Prodgers Aufnahmen ist jedoch weniger eine Leerstelle, die in Erscheinung tritt, wenn das eigentliche Subjekt nicht zu sehen ist - wie in den Kamerafallen, die im Kontext eines Forschungsprojekts zu SaF05 aufgestellt wurden - sie ist vielmehr selbst Subjekt des Films. Die filmische Praxis, die Art und Weise, wie Landschaften in dem Film präsentiert werden, muss dabei als aktive und gezielte Visualisierung des attunement, als ein Vorschlag für einen Umgang mit der Welt (» as a proposition for engaging with the world $\ll)$, als ein verkörpertes Erfassen der Landschaften und Umherfühlen (»feeling around «) verstanden werden, ${ }^{5}$ wie ich in diesem Artikel herausarbeiten werde. Dieses filmische Erfassen geht der Frage nach, was der queere Körper ist und sein kann und wie dieser die Welt navigiert, in der er eingebunden ist.

Prodgers essayistischer, autobiographischer Film besteht aus Indexen, Fragmenten, Fixierungen und Anekdoten, wie Jaclyn Bruneau schreibt, arrangiert wie bewegliche Punkte auf einem Raster ( $\gg$ arranged together like $>$ moving points on a grid $<\ll)^{6}$ und bedient sich einer Anzahl unterschiedlicher Quellen - von Archivmaterial zu Tagebucheinträgen, Korrespondenzen, Journal-Einträgen, Geschichten und Mittel gegen das Vergessen (»correspondences, journal entries, stories, and other defenses against forgetting $\ll){ }^{7}$ In diesem Artikel

5 Dust \& Shadow Reader, 1, hg. v. Maja Kuzmanovic, Nik Gaffney, Ron Broglio, Adam Nocek und Stacey Moran Nocek (März 2018) <https://libarynth.org/dust_and_ shadow/reader_1> [Zugriff: 2. Juni 2021] und Dust \& Shadow Reader, 2, hg. v. Maja Kuzmanovic, Nik Gaffney, Ron Broglio und Adam Nocek (März 2019) <https: //libarynth.org/dust_and_shadow/reader_2> [Zugriff: 2. Juni 2021].

6 Jaclyn Bruneau, $\gg$ New Artist Focus: Jaclyn Bruneau on Charlotte Prodger «, LUX, 23. Juli $2020<$ https://lux.org.uk/writing/new-artist-focus-jaclyn-bruneauon-charlotte-prodger> [Zugriff: 2. Juni 2021].

7 Ebd. 
werde ich dieses Verhältnis zwischen queeren Körpern, queerem Begehren, Landschaften und Technologien, das in den ersten Minuten des Films direkt aufgerufen wird, fokussieren. Ich werde dabei vor allem die verkörperte Wahrnehmung der Landschaften über Technologien, die Situiertheit der Filmemacherin als selbstidentifizierte queere Person in diesen Landschaften und das Einbringen von Erinnerungen an queere Beziehungen in Relation zu dem von Prodger als queer zu lesendem Tierkörper mithilfe der Medien adressieren und der Frage nachgehen, inwiefern die von Prodger entwickelte filmische Methode gleichzeitig eine Orientierung und Desorientierung aufmacht, die in dem Begriff von Queerness bereits angelegt sind. ${ }^{8}$ Dafür werde ich das Konzept der mediated landscapes produktiv machen, das auf die Art und Weise verweist, in der das Verhältnis, die Beziehung zu Landschaften oder der Umgebung (vor allem Orte der Extraktion oder des Traumas) mediatisiert sind und zwar im weitesten Sinne, von deep listening bis zu der Erkundung neuer Visualisierungstechnologien. Der Begriff der mediated landscapes drückt dabei auf zweifache Weise das Verhältnis von Medien und Landschaften aus: zum einen geht es darum, wie Landschaften durch unterschiedliche Medien erst wahrnehmbar und kenntlich gemacht werden (z. B. durch Sensoren, Satelliten und Mobiltelefone), zum anderen, wie Landschaften selbst vermitteln bzw. in und als Medien in Erscheinung treten, und damit selbst in einen Prozess des Wahrnehmens eingebunden sind. ${ }^{9}$

\section{LANDSCHAFTEN UND ANDERE QUEERE RÄUME}

SaF05 wurde für den schottischen Pavillon während der 58. Biennale in Venedig produziert und ist der letzte Film einer Trilogie, die 2015 mit Stoneymollan Trail (Single channel HD video, $43 \mathrm{~min}$ ) begonnen hat, gefolgt von BRIDGIT (Single channel $\mathrm{HD}$ video, $32 \mathrm{~min}$ ), für den Prodger 2018 den Turner Prize gewann. ${ }^{10}$ Die Trilogie markiert insofern eine Veränderung in Prodgers künstlerischer Praxis, als dass sie

8 Siehe dazu auch Sara Ahmed, Queer Phenomenology: Orientations, Objects, Others (Durham, NC: Duke University Press, 2006).

9 Siehe dazu z. B. Jussi Parikka, A Geology of Media (Minneapolis: University of Minnesota Press, 2015) und Benjamin H. Bratton, The Terraforming (Moskau: Strelka, 2019).

10 Stoneymollan Trail, Regie: Charlotte Prodger (Hollybush Gardens, Charlotte Prodger, 2015). 
sich von den vorangegangenen Multi-Monitor-Installationen hin zur Einkanal-Filmprojektion bewegt. Allen drei Filmen gemeinsam sind die Sequenzen langer Totalaufnahmen aus unterschiedlichen Filmarchiven, die Prodger gelagert/gesammelt hat und um die herum sie ihre Filme strukturiert. Über diese legt sie eingesprochene Narrationen, die im Bild keine direkte Entsprechung zu finden scheinen. In Stoneymollan Trail etwa greift sie auf ein Archiv von MiniDV-Kassetten zurück, die in den Jahren zwischen 1999 und 2013 aufgenommen wurden und deren materielle Konstitution sichtbar schlechter wird. In BRIDGIT sind es vor allem iPhone-Aufnahmen, die den Film visuell strukturieren und das Verhältnis zwischen dem (eigenen) Körper und der Technologie noch einmal ganz neu aufmachen. BRIDGIT beginnt mit einer Sequenz, in der Prodger die Kamera auf ihrer Brust, mit Blick auf ihre ausgestreckten Beine, positioniert. Die Kamera bewegt sich mit der Bewegung des Körpers; das Bild ist nie ganz still, genauso wenig wie unsere Körper richtig still sein können - in BRIDGIT wird hier das Verhältnis zwischen den Atembewegungen und der Kamerabewegung sichtbar, wie Bridget Crone schreibt:

The camera moves gently as the body breathes; it is a collaboration that produces a very particular image for here is a body present, embodied yet filmed as if geologic contours; it is at once both the map and its abstraction, the cosmos and a defined set of limits. ${ }^{11}$

Die iPhone-Kamera wird zu einer Verlängerung des Körpers und somit zu einem verkörperten Zeugen des In-der-Welt-Seins, wie Crone argumentiert, ist aber gleichzeitig auch eine Einschränkung in der Bewegung. ${ }^{12}$ In diesem Sinne ist Prodger niemals außerhalb der Aufnahme, wie es Prodger selbst beschreibt:

11 Crone beschreibt damit, in Anlehnung an Nancy Holt und Robert Smithsons Film Swamp (Holt und Smithson, 1971; 16 mm-Film; 6 Min., Farbe, Sound), das Bild als verkörpertes: »Here there is sense of an image that is embodied, inhabited, and produced through the closure of the gap between body (eye) and apparatus (camera). What ensues from this is always a process with rather than for the camera, a process that remakes the limitations of both camera and eye. « Siehe Bridget Crone, »Swampy Ecologies«, Holt/Smithson Foundation, 20. Mai $2020<$ https:// holtsmithsonfoundation.org/swampy-ecologies $>$ [Zugriff: 2. Juni 2021].

Ebd. 
Your fingers get in the shot, they're fleshy, they're right here when they're on the screen and you can see the blood inside your fingers if you cover the tiny lens. You touch that and rub the screen to alter the exposure $[\ldots]$. The systems of the body are enmeshed with the camera. It's a kind of symbiosis but also a kind of grappling. ${ }^{13}$

In SaF05 gibt es darüber hinaus eine längere Sequenz, in der die Spiegelung von Prodgers Laptop-Screen zu sehen ist, markiert von Schmutzflecken und Fingerabdrücken, die dort hinterlassen wurden, als Prodger den Film geschnitten hat. Was Prodger zu interessieren scheint, ist die ständige Transformation audiovisueller Medien, die Veränderung des Filmkörpers, die Veränderung des Formats des bewegten Bildes, dessen Vergänglichkeit, dessen Zeiterfahrung und Zeitlimitationen. So erzählt sie im Zusammenhang mit der Turner Prize-Nominierung, wie erfahrene Kameraleute anhand des Gewichts der 16mm-Filmrolle genau wussten, wieviel Filmzeit auf der Rolle war - während das Gewicht des iPhones nichts über die Speicherkapazität aussagt; die Speicherkapazität von Smartphones allerdings determiniert die mögliche Länge der einzelnen Aufnahmen. ${ }^{14}$

In $\mathrm{SaF} 05$ bleibt Prodger bei der Dokumentation durch das Smartphone, aber kombiniert diese archivierten Aufnahmen mit denen professioneller Kameras, mit Drohnenbildern und den oben erwähnten Kamerafallen; Prodger ist nur eine von fünf Kameraleuten, die im Abspann aufgelistet werden. Low-tech/small handheld devicesAufnahmen wie die vom iPhone, die in bestimmten Momenten an ihre Grenzen kommen (auf der Ebene des Sounds, aber auch der Belichtung), treffen auf High-tech-Aufnahmen wie denen von Drohnen, mit denen ganz andere Machtpolitiken und Blickregime assoziiert werden. Jede Kamera wird für ihre inhärenten materiellen Eigenschaften genutzt. ${ }^{15}$ Dabei spielt Prodger explizit mit den technischen Eigenschaf-

13 »Charlotte Prodger | Turner Prize Winner 2018 | TateShots «, Tate Britain, YouTube, 17. September 2018 <https://www.youtube.com/watch?v=AsVWk5DlbCE.> [Zugriff: 2. Juni 2021].

14 Ebd.

15 »Charlotte Prodger: SaF05 - Scotland + Venice 2019 Official Collateral Event for the 58th International Art Exhibition - La Biennale di Venezia «, Scotland + Venice, 7. Mai $2019<\mathrm{https} / / /$ scotlandandvenice.com/charlotte-prodger-saf05-scotlandvenice-2019- official-collateral-event-for-the-58th-international-art-exhibition-labiennale-di-venezia/> [Zugriff: 2. Juni 2021]. 


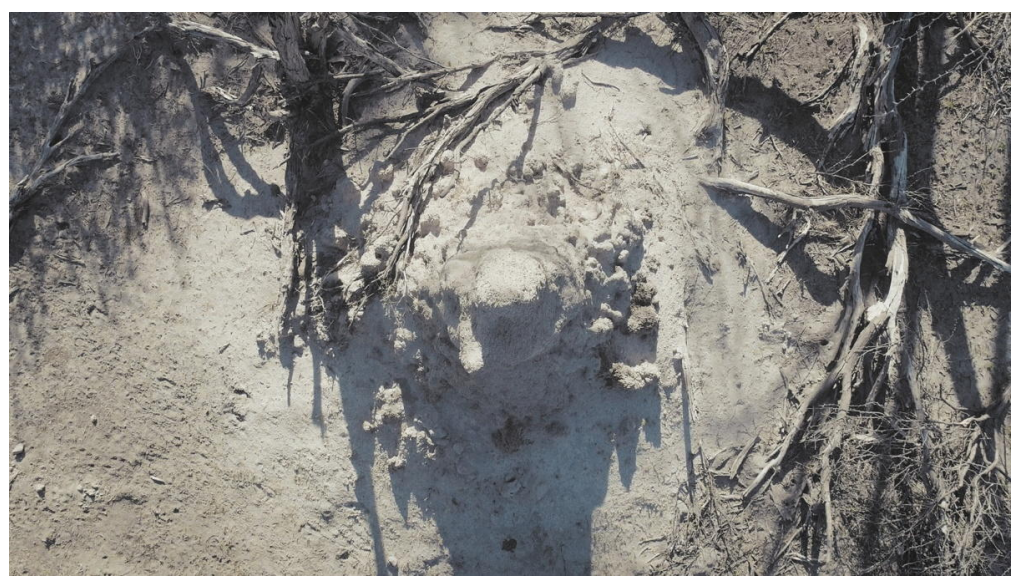

Abb. 4. Film-Still, SaF05, Regie: Charlotte Prodger (Copyright Hollybush Gardens, Charlotte Prodger, 2019).

ten, die den unterschiedlichen Aufnahmemedien zugrunde liegen, und stellt sie in einen queeren Kontext. Die Drohne z. B. wurde zunächst für das Militär entwickelt und von Deutschland in Kriegen wie in Afghanistan und dem Kosovo eingesetzt. In 'Desert Radio Drone' zeigt Ursula Biemann, wie Überwachungsdrohnen auf der Suche nach Migrant*innen im Grenzgebiet des südlichen Libyen eingesetzt werden, $\gg$ transmitting televisual data back to a remote receiver in real time $\ll .{ }^{16}$ Interessanterweise nutzt Prodger die Drohne nicht in diesem Sinne, um sie z. B. in die Suche nach SaF05 einzubinden (und damit die Suche nach Migrant ${ }^{*}$ innen auf die nach der Löwin zu verschieben). Sie nutzt die Drohne allein, um die Termitenhügel zu fokussieren (s. Abb. 4), die sie im Okavango Delta vorfindet und die sie von der Löwin abzulenken scheinen, wie Bruneau schreibt:

$\gg$ Anthropomorphic spectres, analogues of human bodies $\ll$ is what they became to her, soon finding that the magnetic pull she felt to them rivaled the pursuit of SaF05. It's fitting, then,

16 'Desert Radio Drone', in Sahara Chronicle, Regie: Ursula Biemann (Ursula Biemann, 2006-09). Das Zitat (dt.: zur Echtzeit-Übertragung televisueller Daten an einen Funkempfänger) ist direkt aus Biemanns Film genommen; siehe 'Desert Radio Drone', in Sahara Chronicle, Online-Video, Vimeo <https://vimeo.com/534312667>, 16:00-21:40, hier: 16:35, sowie die Beschreibung auf World of Matter <http://www. worldofmatter.net/desert-radio-drone> [Zugriff: 21. September 2021]. 
that each character from her past is given an alphanumeric name comprised of three letters and two numbers, like SaF05's, and that the mounds are used as chapter headings: reminders of the subterranean, the sublimated at each turn. ${ }^{17}$

Wir hören, wie Prodger die Person anleitet, die die Drohne bedient. Mit diesen Drohnenbildern beginnt das zweite Kapitel des Films mit dem Titel $\gg$ FAHRENHEIT $\ll$, der auf eine Temperatureinheit verweist, die sich auf die Temperatur im Termitenhügel selbst oder die hohen Temperaturen der Wüste des Okavango Deltas beziehen könnte. Der Titel verweist gleichzeitig auf ein Parfum, das in der kurz darauf beschriebenen intimen Begegnung mit einer Person in Erscheinung tritt, die sie - in Anlehnung an SaF05 - als BaF89 kodiert.

Im vertikalen Blick auf den Termitenhügel ist die Drohne hörbar und als Schatten sichtbar; sie schwirrt wie ein kleines Insekt links vom Hügel, das heißt, die Sonne steht sehr hoch. Prodger geht es also bei der Aufnahme weniger um das perfekte Bild, das sie in dieser Region vor allem mit dem warmen Licht der frühen Morgen- und Abendstunden einholen würde. Die sanft eingesprochenen Anweisungen von Prodger sind zu hören, die den vertikalen Blick von oben weniger destabilisierend machen: ${ }^{18} \gg[\mathrm{S}]$ tart very slowly moving upward $\ll$, $\gg$ slowly«, »can you hold it? «. In der Bewegung der Drohne weiter nach oben lernen wir mehr von der wüstenartigen Landschaft rund um den Termitenhügel kennen.

Über diese Bilder wird die Erinnerung an eine Begegnung Prodgers mit BaF89 eingesprochen, als sie beide als Reinigungskräfte in Apartments in Barmorrow arbeiteten und zusammen Acid nahmen. Die Erinnerung an eine erotische Begegnung wird über den diegetischen Sound, die gleichzeitig gesprochene Technikanleitung an die Drohnensteuerung, ${ }^{19}$ gelegt und korrespondiert mit dieser, die im Folgenden mit Anführungszeichen gekennzeichnet ist:

17 Bruneau, »New Artist Focus «.

18 Zur Desorientierung in Bezug auf Vertigo und Vertikalität siehe zum Beispiel Hito Steyerl, »In Free Fall: A Thought Experiment on Vertical Perspective «, e-flux journal, 24 (April 2011) <https://www.e-flux.com/journal/24/67860/in-free-fall-athought-experiment-on-vertical-perspective/> [Zugriff: 2. Juni 2021].

19 Prodger ist in ihren Arbeiten von Nancy Holt und Robert Smithson und ihrer Konzeption und Praxis von land art inspiriert. In dem gemeinsamen Film Swamp, Regie: Nancy Holt, Robert Smithson (Holt und Smithson, 1971) läuft Holt mit einer BolexKamera durch die Marschenlandschaft New Jerseys, dicht gefolgt von Smithson, der 


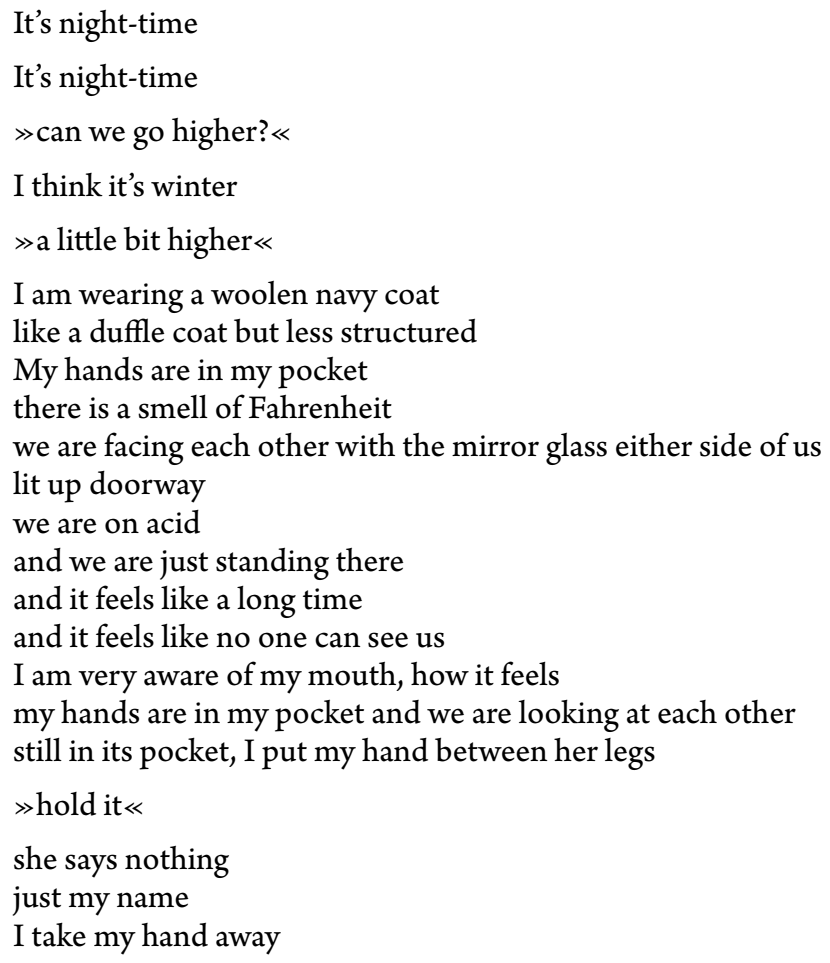

Die Drohnenkamera ist mittlerweile so weit oben, dass die unterschiedlichen Fährten und eine Wasserquelle in der Umgebung des Termitenhügels sichtbar sind. Es folgt ein Schwarzbild, über das das leise Motorengeräusch der Drohne zunächst noch weiter zu hören ist. Dann gibt es auch auf der Ebene des Sounds einen Bruch, Vogelgezwitscher ist nun zu hören und ein Bild erscheint, das ebenerdig und von der Seite einen Termitenhügel fokussiert (s. Abb. 5). Nach dem scaling out der Drohne wechselt Prodger somit zum zooming in und zooming out.

Hier geht es um Rahmungen und Perspektiven, es geht aber auch um das Spannungsverhältnis zwischen Macro und Micro, von Nähe und Distanz, auch zwischen unterschiedlichen geographischen Kontexten sowie der Überlagerung von persönlichen Geschichten, die vorwiegend im Kontext Schottlands verortet sind, und Bildern von

sie anleitet, da ihre Sicht durch den Sucher eingeschränkt ist. Prodger referiert in dieser Drohnenszene auf Smithsons Filmanleitung und überträgt sie auf eine vertikale Ebene sowie in einen explizit queeren Kontext. 


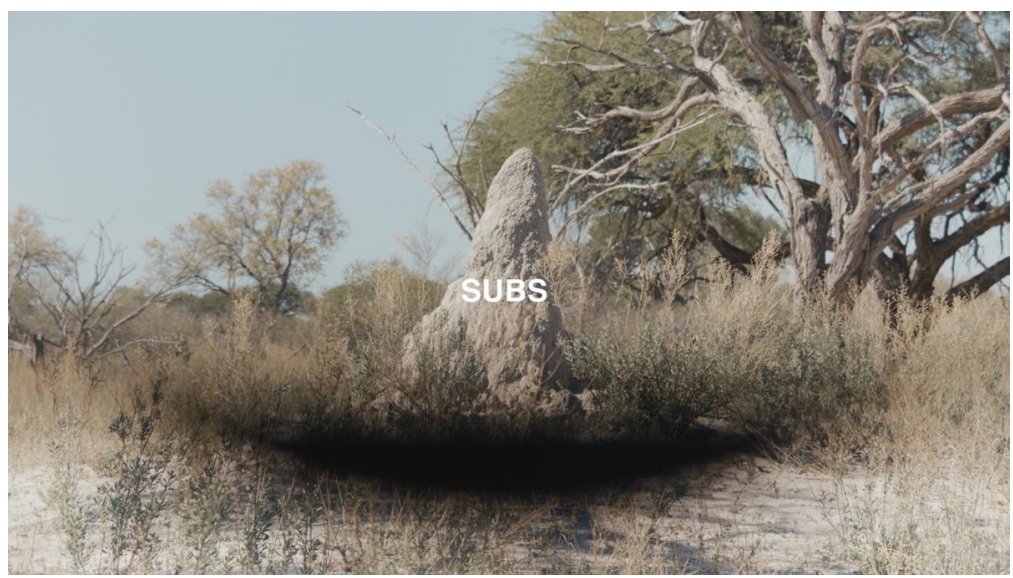

Abb. 5. Film-Still, SaF05, Regie: Charlotte Prodger (Copyright Hollybush Gardens, Charlotte Prodger, 2019).

Botswana und damit dem afrikanischen Kontinent. Und, damit zusammenhängend, geht es um das, was wir sehen, und das, was wir nicht sehen, was vor uns verborgen bleibt. Beide Perspektiven auf den/die Termitenhügel fokussieren die überirdische Struktur; das Kameraauge sieht in diesem Sinne nicht mehr als das menschliche Auge, denn der größte Teil der Termitenhügelstruktur bleibt weiterhin verborgen (was vielleicht mit der extremen Fokussierung von der Seite, in der das Bild an seine Grenzen kommt und verschwimmt, deutlich wird). ${ }^{20}$ Von oben wird die nördlich ausgerichtete Spitze des Hügels fokussiert, der in der Region etwa bei 19 Grad liegt, was mit dem durchschnittlichen Sonnenstand in der Region korrespondiert ${ }^{21}$ und auf Prodgers Entscheidung verweist, die Drohnenaufnahme dann zu machen, wenn die Sonne am höchsten steht. Wir sehen von den unterschiedlichen

20 Zum Inneren des Termitenhügels, also dem Teil, der unter der Erdoberfläche angelegt ist, heißt es in der Forschung: $\gg$ Inside the mound is an extensive reticulum of tunnels and conduits, which reveals its function: the mound is an organ of physiology for the termite colony superorganism, which is centered on the underground nest. The nest itself is a spheroidal structure consisting of numerous gallery chambers, each of which contains a fungus comb, where raw forage, such as grass and wood, is digested by the symbiotic fungi (Termitomyces) that the termites cultivate within their nest.« Siehe »Structure of the Termite Mound «, Termite Research <https://www.esf.edu/ efb/turner/termitePages/termiteStruct.html\#intArch> [Zugriff: 2. Juni 2021]. Ebd. 
Blickrichtungen auf den Termitenhügel nur den kleinsten Teil von dessen Struktur - nur die Oberfläche der oberirdischen Struktur, die vorwiegend leer und unbewohnt ist. Termiten, die sich in diesem Bereich aufhalten, verteidigen entweder die Kolonie oder reparieren die Struktur, was vor allem nach Regenfällen (während der Regenzeit, also saisonal) der Fall ist. ${ }^{22}$ Was wir gerade in der Seitenansicht sehen (dem zooming in, bevor das Bild undeutlich wird), ist diese Art der reparativen Arbeit, die aufzeigt, wie dynamisch und porös die Oberfläche der Termitenhügel ist: $\gg$ As soil is eroded from the actions of wind and rain, it is replaced by the action of termites depositing fresh soil. This makes the mound structure malleable, which allows it to be modeled to local conditions. $\ll{ }^{23}$ Durch diese neuen Ablagerungen auf der Oberfläche kann Wind dringen, der direkt in das Tunnelsystem des Termitenhügels strömt und zur Ventilation beiträgt. Durch das zooming in wird die Oberflächenstruktur in den Blick genommen und damit die Materialität des Hügels, die vorwiegend aus Quarzpartikeln besteht und mit einer Mixtur aus Speichel und Lehm verfestigt und verbunden ist.

Im Inneren des Termitenhügels - in dem Teil, den wir nicht sehen (aber auf den in einem der folgenden Untertitel als $\gg$ TUNNELS $\ll$ referiert wird) - befinden sich die zur Ventilation und Zirkulation von Luft angelegten Tunnel. Termiten leben in großen Kolonien und müssen ein bestimmtes Level an Temperatur (bis zu 30 Grad bzw. 86 Grad Fahrenheit), Feuchtigkeit und respiratorische Gase wie Kohlendioxid aufrechterhalten. Dazu gehen die Termiten in einigen Regionen eine Symbiose mit bestimmten Pilzen ein (in Namibia und Botswana sind es die Omajova-Pilze). Die Pilze bekommen Nahrung, Wasser und Schutz von den Termiten im Austausch für deren Verarbeitung der Zellulose. Wärme wird sowohl von den Pilzen als auch von den Termiten produziert. ${ }^{24}$ Die Idee der Symbiose als Ermöglichung, die Prodger bereits in Bezug auf das iPhone und den eigenen Körper angesprochen

$22 \gg$ The turnover of soil in the mound is seasonal, occurring solely during the rainy season. It is also quite prodigious, amounting to about $250 \mathrm{~kg}$ dry mass of soil annually. It is for this reason that these mounds have been likened to slow-motion $>$ soil fountains $<$. $\ll$ Siehe ebd.

23 Ebd.

24 Stephanie L. Richards, »Termite Mound Structures«, Terminix <https://www. terminix.com/termite-control/colonies/termite-mounds> [Zugriff: 2. Juni 2021]. 
und visualisiert hat, wird hier noch einmal anders thematisiert und auf das nicht-menschliche, komplexe Netzwerk zwischen Termiten und Pilzen, aber auch Bäumen, erweitert - auch in Bezug auf die Energieeffizienz (in Bezug auf externe und interne Struktur, Wetter und Environment, und die Fähigkeit, die Temperatur der internen Struktur $\mathrm{zu}$ regulieren): $\gg$ The results indicate that the mound superstructure and internal condition strongly depend on the combined effect of environmental forces. $\ll 25$

In der Verbindung von den Drohnenbildern der komplexen Termitenhügelstrukturen, der verbalen Anleitung der Drohne und der Narration queeren Begehrens werden die Machtpolitiken, die mit der mobilen Technologie verbunden sind, unterwandert. In dieser Unterwanderung weigert sich Prodger, einen kolonialen Blick zu reproduzieren, der mit »leeren « Landschaftsaufnahmen auf dem afrikanischen Kontinent eng verbunden ist und durch den $u$. a. der Mythos des leeren Raums verortet wurde, die Idee der terra nullius. ${ }^{26}$ In SaF05 sehen wir keine Menschen, aber wir hören sie, sie sind somit im Bild - das wird auch in der letzten Szene des Films deutlich, in der Prodger diejenige Person anleitet, die die Drohne lenkt: »Okay, bring it down very slowly and keep it down all the way«. Gleichzeitig hören wir eine Funkkommunikation zwischen Bajaki Jacks Amos vom BPCT, der bei Prodger und ihrer Filmcrew ist, und einem anderen Suchtrupp, der einer möglichen Spur von SaF05 weiter folgt. Es sind die letzten Stunden, die Prodger in Botswana bleiben. Während also die zweite Gruppe die verbleibende Zeit nutzt, SaF05 zu orten, zoomen Prodger und ihre Gruppe auf den Termitenhügel - die Drohne bleibt über der Spitze schweben, während wir das Geräusch hören, das auf die leere Batterie aufmerksam macht, gefolgt von einem Schwarzbild; das Bat-

25 Tadeu Mendonca Fagundes, Juan Carlos Ordonez und Neda Yaghoobian, $\gg$ How the Thermal Environment Shapes the Structure of Termite Mounds $\ll$, Royal Society Open Science, 7.1 (2020) <https://doi.org/10.1098/rsos.191332>.

26 Dieser Diskurs diente unter anderem zur Rechtfertigung der Landnahme bzw. Landenteignung in den Kolonien und hat bis heute in vielen ehemaligen Kolonien Implikationen für die Landfrage und die Frage der Restitution - denn die Idee, da habe keiner gelebt, also sei die Fruchtbarmachung des brach liegenden Landes nicht nur wichtig gewesen, sondern habe auch niemandem Land weggenommen, bedeutet, es müsse auch nicht zurückgegeben werden. Für weitere Informationen zu dem Verhältnis von Landschaft und Kolonialismus siehe z. B. Renzo Baas, Fictioning Nambia as a Space of Desire (Basel: Basler Afrika Bibliographien, 2019) und Nicola Brandt, Landscapes: Between Then and Now (London: Bloomsbury, 2020). 
teriegeräusch ist noch ein wenig zu hören, bis auch der Sound ausgeht, the end.

\section{CODES, RASTER UND VERKÖRPERTE FILMPRAKTIKEN}

Das Ende bringt die Denkfigur SaF05, die für Prodger für queere Subjektivität steht, mit der kollektiven Struktur, dem Schwarmbewusstsein (hive mind) der Termiten zusammen. Wie am Anfang des Films wird auch am Ende die Landschaft als immer schon medial vermittelt verstanden. Hier werden die verschiedenen Kameras dazu verwendet, Methoden und Politiken der Wahrnehmung und Orientierung sichtbar zu machen, der Berührung, der Bewegung und des Stillhaltens, die durch die unterschiedlichen Filmkörper möglich sind bzw. ermöglicht werden. Was Prodger mit den verschiedenen Technologien macht, wie sie sich mit der Kamera bewegt und wie sie beobachtet, wie sie das Verhältnis von Nähe und Distanz navigiert bzw. das Verhältnis zwischen Konzentrieren und Desorientieren (Wackeln, schnelles Gehen, Unschärfe durch zu große Nähe versus das genaue Fokussieren, dem Verweilen an einer Gesteinsformation, teilweise sinnierend, kontemplierend; manchmal kontrolliert, manchmal weniger), muss als eine komplexe Methodologie der verkörperten Wahrnehmung und Orientierung verstanden werden, die die Aufmerksamkeit hin auf Elemente, Rhythmen und Kräfte wendet, die am Rande unserer Erfahrung existieren. Anna Tsing spricht in diesem Zusammenhang von den »arts of noticing ${ }^{27}$ und W. J. T. Mitchell in Landscape and Power von einer Verschiebung vom Nomen »landscape « hin zum Verb »landscaping «: um danach zu fragen, wie Landschaft gedacht werden kann, nicht als ein zu betrachtendes Objekt oder ein zu lesender Text, sondern als ein Prozess, durch den soziale und subjektive Identitäten geformt werden ( $\gg[$ to] ask that we think of landscape, not as an object to be seen or a text to be read, but as a process by which social and subjective identities are formed $\ll){ }^{28}$

27 Anna Tsing, The Mushroom at the End of the World: On the Possibility of Life in Capitalist Ruins (Princeton, NJ: Princeton University Press, 2015), S. 37.

28 W. J. T. Mitchell, Landscape and Power (Chicago: University of Chicago Press, 2002 [1994]), S. 1. In ihrem Text $\gg$ Necrolandscaping « nimmt Jill Casid diese Verschiebung schließlich in einem explizit queeren Kontext vor, insbesondere in Bezug auf 
Prodgers »embodied sensing « mithilfe der Kamera unterscheidet sich also fundamental von der Geschichte der Landschaftsdarstellung, die in der Landschaftsmalerei vielleicht ihren Ursprung hat und die Mitchell kontextualisiert. In SaF05 haben wir es mit filmischen Methoden der visuellen Kontemplation zu tun (wie in der Geschichte der Landschaftsmalerei angelegt), und - ganz in der Tradition, neue optische Technologien in der Landschaftsdarstellung einzusetzen ${ }^{29}$ werden sie auch hier als visuelle Aneignung und Fokus der Identitätsformation ( $\gg$ site of visual appropriation, a focus for the formation of identity $\ll$ ) verhandelt, wie Mitchell schreibt. ${ }^{30}$ Und weiter: »landscape is a dynamic medium, in which we >live and move and have our being, $<$ but also a medium that is itself in motion from one place or time to another « (Landschaft ist ein dynamisches Medium, in dem wir leben, uns bewegen und sind; aber auch ein Medium, das selbst in räumlicher und zeitlicher Bewegung ist). ${ }^{31}$

Mit der Verschiebung auf das Prozessuale geht Prodger über die Subjekt-Objekt-Spaltung hinaus, die in klassischen Landschaftsdarstellungen angelegt ist und die eine bestimmte Blickperspektive beinhaltet. Ähnlich wie andere gegenwärtige Künstler*innen ${ }^{32}$ desta-

Tod/Verlust durch Gewalt gegen Trans* Personen. Siehe Jill H. Casid, »Necrolandscaping «, in Natura: Environmental Aesthetics after Landscape, hg. v. Jens Andermann, Lisa Blackmore und Dayron Carrillo Morell (Zürich: Diaphanes, 2018), S. 237-64. Prodger selbst thematisiert das Konzept von landscapes folgendermaßen: »I feel very cautious about the term landscape as one thing, like a homogenous romantic blob, like a forest or a mountain as opposed to cities which are generally more individuated. People see a shot of a city in a film I feel like often as an impulse to try to identify that space. Where is that? Is that Paris? Which cross-section is that? So rather than depicting landscape generically, I try to foreground subjectivity like in the form of personal voiceover to explore landscapes as the distinct individual locations that they are.«Zit. aus »Charlotte Prodger | Turner Prize Winner 2018 | TateShots «.

29 Svetlana Alpers beschreibt z. B., wie die Techniken des Linsenschleifens und der Einsatz diverser Geräte mit den neu erfundenen Linsen im 17. Jahrhundert in den Niederlanden zu neuen Landschaftsbildern führten, sowohl in Maltechniken/Perspektiven, als auch in den Bedeutungszuschreibungen z. B. von Himmel und Horizont. Vgl. Svetlana Alpers, Kunst als Beschreibung. Holländische Malerei des 17. Jahrhunderts, übers. v. Hans Udo Davitt (Köln: Dumont, 1985).

30 Mitchell, Landscape and Power, S. 2.

31 Ebd.

32 Siehe z. B. das Projekt Geocinema (2018) von Asia Bazdyrieva, Alexey Orlov and Solveig Suess <https://www.geocinema.network/> [Zugriff: 2. Juni 2021] sowie die Arbeiten von Ignacio Acosta, Copper Geographies (2010-2016) <http: //ignacioacosta.com/copper-geographies> [Zugriff: 2. Juni 2021]; Sam Nightingale, »Photochemical Alchemy in Sub-Arctic Finland», samnightingale.com, 
bilisiert Prodger somit bestimmte Landschaftskonventionen, um die komplexe technologische geopolitische Situation, in der wir uns befinden, auszudrücken und mit ihren eigenen, ganz persönlichen queeren Erfahrungen zu verbinden. Damit zeigt sie, dass Landschaften (und Identitäten?) fragmentiert und porös sind, unbegrenzt, unstabil - und als eine Art expansives Netzwerk von widersprüchlichen, aber verbundenen Systemen verstanden werden müssen, die es zu erkunden und für sich selbst erfahrbar zu machen gilt:

Each work reads as though Charlotte is using the tip of her finger to slowly, sedulously trace a thing's contours, every part of it - not so much to comprehend it, but to sustain proximity, to think of nothing else, to nurture its association, and then trace them too. ${ }^{33}$

Dieser verkörperte Umgang mit der Kamera als eine filmische Methode des attunements verweist auf den Prozess, den Crone als $\gg$ a process with rather than for the camera $\ll$ beschreibt, ${ }^{34}$ eine intensive körperliche Erfahrung von Anwesendsein (»of being present «), und gleichzeitig eine Expansion der eigenen sensorischen Welt durch diese Erfahrung. Landschaften werden uns somit als multidimensionale Relationen zwischen Körpern und Daten und anderen Formen von Leben und Materialien präsentiert. ${ }^{35}$

In BRIDGIT hat Prodger das Verhältnis zwischen Daten und Landschaften nicht zuletzt über das Raster, das sie über einen Menhir gelegt hat, dargestellt. Dieses Raster taucht auch in SaF05 wieder auf

17. Mai 2019 <https://www.samnightingale.com/photochemical-alchemy-in-subarctic-finland/> [Zugriff: 2. Juni 2021] und Joshua Bonnettas Lago (2015) <http: //shelter-press.org/sp069-joshua-bonnetta-lago/> [Zugriff: 2. Juni 2021].

33 Bruneau, »New Artist Focus «.

34 Bridget Crone, $\gg$ Swampy Ecologies $\ll$.

35 Jussi Parikka begreift dies (in Anlehnung an Donna Haraways Konzeption von naturecultures) als medianatures. So argumentiert er: $\gg[t]$ he ties are intensively connected in material non-human realities as much as in relations, economy and work «; siehe Parikka, A Geology of Media, S. 14. Geologie, Natur und Medien kommen hier also zusammen. Laut Parikka sind diese Elemente untrennbar miteinander verbunden, denn wenn es um Medien geht, geht es immer auch um Mineralien und um chemische Komponenten. Diese sind wiederum untrennbar von den prekären Arbeitsbedingungen und der Ausbeutung von Arbeiter*innen in Minen, die die Mineralien bergen. Sie sind aber auch untrennbar von denjenigen, die in den Fabriken arbeiten und Komponenten der iPhones produzieren, die oft in Umgebungen und Bedingungen arbeiten, die prekär, nicht sicher sind oder umgeben von Materialien, die einen schädlichen Effekt auf die Gesundheit haben. 
- und zwar in der Arbeit mit Bildern von assyrischen Palastreliefs, die Löwenjagden darstellen und ursprünglich dreidimensionale Basreliefs sind:

I'd always been blown away by these Assyrian reliefs, particularly in terms of perspective. So, for example, to show that one figure is behind another figure, in the background, rather than making it smaller than the figure in the foreground, it's put on top of the figure in the foreground to show there's distance between them. [...] I found these photographs in a book and I pulled some of them out and I started playing around with them on a cutting matt that I had in my studio, making collages. [...] The cutting matt is gridded. [...] I think about grids a lot, particularly in relation to coordinates. So, grids are used to map things, they contain and frame things. But in the end, grids are always infinite. What we can't see of them extends infinitely beyond the edges of the frame. And for me this feels very vast, like lot of the empty landscapes I am drawn to in my work. ${ }^{36}$

Was Prodger also konsequent in ihren Arbeiten betreibt ist die den Technologien inhärenten Möglichkeiten herauszufordern und in neuen Konstellationen produktiv zu machen. Somit wird das Raster, das nach Dimitris Papadopolous, Niamh Stephenson und Vassilis Tsianos unwahrnehmbare Körper und Subjektivitäten in Subjekte transformiert (und in Gruppierungen und Territorien einteilt) ${ }^{37}$ gleichzeitig zu einer Ermöglichung, über queere Körper, Landschaften und Transformation nachzudenken - nicht nur in Bezug auf den Tierkörper, sondern auch in Bezug auf das eigene Selbst. Dabei führen die Überlagerung der persönlichen Geschichte und eigener Bilder mit den Bildern von SaF05 und von Botswana, die Effekte dieser Montage weniger zu einer Einschreibung oder Projektion der Sprechenden, die in den Landschaften ihren Erinnerungen nachhängt, sondern vielmehr zu einer Suche nach (queeren) Koinzidenzen.

Diese Form der Beobachtung des Selbst über Materialität, Verbindungslinien und Analogien spitzt Prodger in $\mathrm{SaFO5}$ beim Vorlesen der Logbucheinträge zu, die im Rahmen eines Forschungsprojekts zu SaF05 angelegt wurden, und u. a. Bewegungsdaten und Verhalten

36 Charlotte Prodger on her Series Palace Prints.

37 Dimitris Papadopolous, Niamh Stephenson und Vassilis Tsianos, Escape Routes: Control and Subversion in the Twenty-First Century (London: Pluto Press, 2008), S. 10. 
dokumentieren, und die Prodger mit eigenen Tagebucheinträgen zu intimen Begegnungen und Erfahrungen von Verlust, wie etwa den Tod von DuF96, zusammenbringt. In einer Sequenz, eine DashcamAufnahme auf der Straße zu Nancy Holts Skulptur Sun Tunnels im ländlichen Utah z. B., ${ }^{38}$ hören wir Prodger unermüdlich und rhythmisch Einträge aus dem Logbuch vorlesen, vermischt mit einer sexuellen Erinnerung an GaF96 - eine Sequenz im Film, die die multidimensionalen Relationen zwischen Körpern, Codes und Daten noch einmal im Bild von Landschaften zusammenbringt und gleichzeitig die Frage aufmacht (ohne sie schlussendlich zu beantworten), was der (queere) Körper in einer Welt sein kann, die aus komplexen Verbindungslinien besteht:

Date: 16th November 2015. Start Time: 17:49. Location: -19.53368357 23.64108922 938.0 5.0. Behaviour: Approach. Behaviour time: 18:17. Who: SaF05. Habitat: Acacia scrub. Recipient ID: Woody. Outcome social: Rubs. Comments: SaF05 presenting. Count: 3. End time: 19:50.

Date: 21 st November 2015. Start Time: 05:55. Location: -19.52420401 23.63942514 940.0 5.0. Behaviour: Grooming. Behaviour time: 06:59. Who: SaF05. Habitat: Acacia scrub. Recipient ID: SaF03. Outcome social: reciprocate. Count: 2. End time: 09:23.

GaF93 was training to be a radiographer. We met at Club $\mathrm{X}$. She pushed me against the wall in a doorway somewhere around Renfrew Street. I've looked for it since when I've been in that area. But I don't know where it was because it was nighttime 27 years ago.

In daylight lying on her bed up against the window in her room inside the student halls in Paisley [GLASGOW] I looked down at the dark triangle of her pubic hair for the first time. I thought what I was seeing was her underwear $[\ldots]$ then realized her underwear wasn't on anymore.

[...]

Date: 21st November 2015. Start Time: 05:55. Location: -19.52420401 23.63942514 940.0 5.0. Behaviour: Moving. Behaviour time: 08:30. Who: SaF05. Habitat: Acacia scrub. Count: 2. End time: 09:23.

38 Nancy Holt, Sun Tunnels, 1973-76, Installation, Beton-Zylinder, Great Basin Wüste, Utah. 
Daniel Kulle beschreibt den Einsatz der eigenen Stimme im queeren Experimentalfilm zu Bildern, die mit dem eingesprochenen Narrativ nicht korrespondieren, als einen autobiographischen Marker, der sich als $\gg$ (provisorischer) Selbstfindungs- und Individuationsprozess lesen lässt $\ll$ :

Die Stimme dient dabei sowohl dazu, eine eigenständige Position als minoritäres Individuum zu finden, als auch dazu, die etablierten Repräsentationen und die Hierarchie der Bilder, denen sie sich gegenüber sieht, zu unterwandern. Durch den Kontrast zwischen Bildwelt und Voice-over wird der vermeintlich unproblematische Individuationsprozess, den die Stimme performativ behauptet, jedoch gleichzeitig widerrufen. Das queere Selbst, das diese Filme postulieren, ist ein notwendigerweise ironisches und metasubjektives: Indem das Selbst sich aus der Reibung an den Bildwelten heraus entwickelt, die Differenz zu diesen aber niemals überwinden kann, bleibt es als Konstrukt ohne Fundament, ein reines Provisorium. Im häufigsten Fall kontrastieren derartige akusmatische Filme die eigene Stimme mit Stadt-, Kultur- oder Naturlandschaften $[\ldots] .{ }^{39}$

In dem Sinne des von Kulle beschriebenen provisorischen, also nichtdefinitiven, nicht-abgeschlossenen Selbstfindungsprozesses verstehe ich auch Prodgers Umgang mit den alphanumerischen Codes, die sie in Anlehnung an SaF05 jeder Person aus ihrer Vergangenheit gibt, die sie in SaF05 erwähnt (was die genannten Personen gleichzeitig anonymisiert). Diese Analogisierung des eigenen Lebens nach den Maßgaben einer biologischen Studie verspricht somit weniger Gewissheit (wie in wissenschaftlichen Studien vermeintlich angelegt), sondern ist vielmehr als eine diffuse Artikulierung queerer Subjektivität und queerer Bindungen zu verstehen, wie Bruneau es beschreibt, und in dem Sinne als ein weiteres Suchen nach Antworten auf die Fragen, was Queerness ausmacht, was queere Körper ausmachen, welche Rolle Geschlechterkategorien dabei spielen und wie man diesen Fragen in einem komplexen Netzwerk von verbundenen Systemen, die über geopolitische Grenzen hinweg angelegt sind, auch ästhetisch näherkommen kann.

39 Daniel Kulle, »Innovation an den Rändern des Queer Cinema. Ästhetische Strategien des Queeren Experimentalfilms «, in Queer Cinema, hg. v. Dagmar Brunow und Simon Dickel (Mainz: Ventil, 2018), S. 226-44, hier S. 234-35. 
Henriette Gunkel, >Codes, Raster, Technologien queerer Erinnerungslandschaften. Charlotte Prodgers $\mathrm{SaFO5} \ll$, in Queeres Kino / Queere Ästhetiken als Dokumentationen des Prekären, hg. v. Astrid Deuber-Mankowsky und Philipp Hanke, Cultural Inquiry, 22 (Berlin: ICI Berlin Press, 2021), S. 97-117<https: //doi.org/10.37050/ci-22_05>

\section{QUELLENANGABEN}

\section{BIBLIOGRAFIE}

Ahmed, Sara, Queer Phenomenology: Orientations, Objects, Others (Durham, NC: Duke University Press, 2006) <https://doi.org/10.1515/9780822388074>

Alpers, Svetlana, Kunst als Beschreibung. Holländische Malerei des 17. Jahrhunderts, übers. v. Hans Udo Davitt (Köln: Dumont, 1985)

Anonym [LUX], »SaF05«, Künstler*innenagentur LUX Scotland (2019) <https://lux.org. uk/work/saf05> [Zugriff: 2. Juni 2021]

Bratton, Benjamin H., The Terraforming (Moskau: Strelka, 2019)

Bruneau, Jaclyn, »New Artist Focus: Jaclyn Bruneau on Charlotte Prodger $\ll$, LUX, 23. Juli $2020<$ https://lux.org.uk/writing/new-artist-focus-jaclyn-bruneau-on-charlotteprodger $>$ [Zugriff: 2. Juni 2021]

Casid, Jill H., »Necrolandscaping «, in Natura: Environmental Aesthetics after Landscape, hg. v. Jens Andermann, Lisa Blackmore und Dayron Carrillo Morell (Zürich: Diaphanes, 2018), S. 237-64

Crone, Bridget, »Swampy Ecologies «, Holt/Smithson Foundation, 20. Mai $2020<$ https: //holtsmithsonfoundation.org/swampy-ecologies> [Zugriff: 2. Juni 2021]

Kulle, Daniel, »Innovation an den Rändern des Queer Cinema. Ästhetische Strategien des Queeren Experimentalfilms «, in Queer Cinema, hg. v. Dagmar Brunow und Simon Dickel (Mainz: Ventil, 2018), S. 226-44

Kuzmanovic, Maja, Nik Gaffney, Ron Broglio und Adam Nocek (Hg.), Dust \& Shadow Reader, 2 (März 2019) <https://libarynth.org/dust_and_shadow/reader_2> [Zugriff: 2. Juni 2021]

Kuzmanovic, Maja, Nik Gaffney, Ron Broglio, Adam Nocek und Stacey Moran Nocek (Hg.), Dust \& Shadow Reader, 1 (März 2018) <https://libarynth.org/dust_and_shadow/ reader_1> [Zugriff: 2. Juni 2021]

Mitchell, W. J. T., Landscape and Power (Chicago: University of Chicago Press, 2002)

Papadopolous, Dimitris, Niamh Stephenson und Vassilis Tsianos, Escape Routes: Control and Subversion in the Twenty-First Century (London: Pluto Press, 2008)

Parikka, Jussi, A Geology of Media (Minneapolis: University of Minnesota Press, 2015) <https: //doi.org/10.5749/minnesota/9780816695515.001.0001> 
Richards, Stephanie L., »Termite Mound Structures «, terminix <https: / /www.terminix.com/ termite-control/colonies/termite-mounds $>$ [Zugriff: 2. Juni 2021]

Tate Britain, »Charlotte Prodger | Turner Prize Winner $2018 \mid$ TateShots «, YouTube, 17. September 2018 <https://www.youtube.com/watch?v=AsVWk5DlbCE> [Zugriff: 3. Juni 2021]

Termite Research, »Structure of the Termite Mound «<https://www.esf.edu/efb/turner/ termitePages/termiteStruct.html $>$ [Zugriff: 2. Juni 2021]

Tsing, Anna, The Mushroom at the End of the World: On the Possibility of Life in Capitalist Ruins (Princeton, NJ: Princeton University Press, 2015) <https://doi.org/10.1515/ 9781400873548>

Young, Linsey, »Hidden in Plain Sight $\ll$, in SaF05: Charlotte Prodger (Cove: Cove Park, Scotland + Venice, 2019), S. 11-17

\section{FILMOGRAFIE}

BRIDGIT, Regie: Charlotte Prodger (Hollybush Gardens, Charlotte Prodger, 2016)

Charlotte Prodger on her Series Palace Prints (Hollybush Gardens, 2019) <https://www. instagram.com/tv/B_qA9M8FtCJ/> [Zugriff: 2. Juni 2021]

SaF05, Regie: Charlotte Prodger (Hollybush Gardens, Charlotte Prodger, 2019)

Sahara Chronicle, Regie: Ursula Biemann (Ursula Biemann, 2006-09)

Stoneymollan Trail, Regie: Charlotte Prodger (Hollybush Gardens, Charlotte Prodger, 2015)

Swamp, Regie: Nancy Holt, Robert Smithson (Holt und Smithson, 1971) 